\title{
Diffusion Tensor Imaging Demonstrates Brainstem and Cerebellar Abnormalities in Congenital Central Hypoventilation Syndrome
}

\author{
RAJESH KUMAR, PAUL M. MACEY, MARY A. WOO, JEFFRY R. ALGER, AND RONALD M. HARPER
}

\author{
Department of Neurobiology [R.K., R.M.H.], Department of Neurology [J.R.A.], Brain Research Institute [P.M.M., R.M.H.], School of \\ Nursing [P.M.M., M.A.W.], University of California at Los Angeles, Los Angeles, California 90095
}

\begin{abstract}
Congenital central hypoventilation syndrome (CCHS) patients show reduced breathing drive during sleep, decreased hypoxic and hypercapnic ventilatory responses, and autonomic and affective deficits, suggesting both brainstem and forebrain injuries. Forebrain damage was previously described in CCHS, but methodological limitations precluded detection of brainstem injury, a concern because genetic mutations in CCHS target brainstem autonomic nuclei. To assess brainstem and cerebellar areas, we used diffusion tensor imaging-based measures, namely axial diffusivity, reflecting water diffusion parallel to fibers, and sensitive to axonal injury, and radial diffusivity, measuring diffusion perpendicular to fibers, and indicative of myelin injury. Diffusion tensor imaging was performed in 12 CCHS and 26 controls, and axial and radial diffusivity maps were compared between groups using analysis of covariance (covariates; age and gender). Increased axial diffusivity in CCHS appeared within the lateral medulla and clusters with injury extended from the dorsal midbrain through the periaqueductal gray, raphé, and superior cerebellar decussation, ventrally to the basal-pons. Cerebellar cortex and deep nuclei, and the superior and inferior cerebellar peduncles showed increased radial diffusivity. Midbrain, pontine, and lateral medullary structures, and the cerebellum and its fiber systems are injured in CCHS, likely contributing to the characteristics found in the syndrome. (Pediatr Res 64: 275-280, 2008)
\end{abstract}

$\mathrm{C}$ ongenital central hypoventilation syndrome (CCHS) is a developmental condition characterized by a loss of the drive to breathe during sleep, reduced ventilatory sensitivity to $\mathrm{CO}_{2}$ and $\mathrm{O}_{2}$, and multiple thermoregulatory, affective, and autonomic deficiencies (1-3). The affective and thermoregulatory functions depend on more-rostral brain sites, such as the hypothalamus and limbic regions, and these areas show injury with quantitative magnetic resonance imaging techniques, such as T2 relaxometry and diffusion tensor imaging (DTI)based mean diffusivity measures $(4,5)$.

Although several physiologic and emotional deficits in CCHS result from rostral brain injury, other breathing and autonomic problems, such as diminished ventilatory sensitivity to $\mathrm{CO}_{2}$ and parasympathetic deficits implicate brainstem involvement. Mutations of PHOX2B, suggested to underlie

Received January 2, 2008; accepted April 23, 2008.

Correspondence: Ronald M. Harper, Ph.D., Department of Neurobiology, David Geffen School of Medicine at UCLA, University of California at Los Angeles, Los Angeles, CA 90095-1763; e-mail: rharper@ucla.edu

Supported by the National Institute of Child Health and Human Development R01 HD-22695. the initial developmental injury in CCHS (6), primarily target autonomic ganglia, which lie in peripheral and brainstem sites, and may result in cellular and axonal deficits. However, previously used MRI procedures, which demonstrate injury to rostral brain areas, show little evidence of expected brainstem injury. Technical limitations associated with spatial resolution and sensitivity have precluded detection of brainstem injury. Thus, injury to much of the reflex circuitry for breathing and parasympathetic motor neuron action that lies in brainstem and cerebellar sites $(7,8)$ have not been adequately assessed. These regions do, however, show functional MR deficits to ventilatory and cold pressor challenges in CCHS subjects $(9-11)$.

Among MRI techniques, DTI is a procedure that measures directional diffusivity of water molecules. Although several DTI-based indices, including mean-diffusivity, can detect brain tissue injury, and show rostral brain damage and some degree of brainstem injury in CCHS (4), these indices are not specific to different types of underlying pathology, and may be insufficiently sensitive to reveal subtle tissue changes. Specialized DTI indices, such as axial diffusivity, a measure of diffusivity parallel to axons, and radial diffusivity, a measure of diffusivity perpendicular to fibers (12), provide markedly enhanced sensitivity to tissue injury. Animal studies infer that axial diffusivity abnormalities accompany axonal injury, while radial diffusivity changes appear with altered myelin (12-14), and both measures are more sensitive compared with other DTI-based indices.

The objective was to evaluate, using axial and radial diffusivity measures, the extent of injury in brainstem and cerebellar sites in CCHS, and determine whether damage occurs in sites contributing to syndrome characteristics.

\section{METHODS}

Subjects. We studied 12 CCHS subjects (mean age \pm SD: $15.2 \pm 2.4$ y; range: $12-18 \mathrm{y} ; 8$ male) and 26 control subjects $(15.5 \pm 2.4 \mathrm{y} ; 10-19 \mathrm{y} ; 15$ male). CCHS subjects were diagnosed based on standard criteria (3), and recruited through the CCHS family network (http://www.cchsnetwork.org). Of 12 CCHS subjects, four showed evidence of PHOX2B mutation, two were inconclusive, and six subjects were never tested. Some CCHS patients require continuous ventilatory support during sleep and waking; we included only cases requiring breathing assistance during sleep. CCHS subjects with additional conditions, such as cardiovascular or neurologic disorders, which can

Abbreviations: CCHS, Congenital central hypoventilation syndrome; DTI, Diffusion tensor imaging; NTS, Nucleus of the solitary tract 
also affect brain tissue structure (e.g., loss of hippocampal tissue in temporal lobe epilepsy), were excluded. CCHS subjects with Hirschsprung's disease were also excluded to avoid concomitant neural injury from intestinal malabsorption, e.g., thiamine deficiency. Control subjects were recruited through advertisements at the university campus, which has a diverse ethnic population; the aim was to target parents who could enroll their children. All studies were performed without anesthesia or sedatives; subjects were conscious throughout, and were given breaks from the scanner if they became restless.

All subjects and their parents/guardians provided informed written consent/assent, and the procedures were approved by the Institutional Review Board of the University of California at Los Angeles.

Magnetic resonance imaging. Brain MRI studies were performed using a 3.0-Tesla MRI scanner (Magnetom Trio; Siemens, Erlangen, Germany), with a whole-body transmitter coil, and a receive-only 8-channel phased-array head-coil. Foam pads were placed on both sides of the head to minimize head motion. High-resolution T1-weighted imaging was performed using a magnetization-prepared-rapid-acquisition-gradient-echo pulse sequence [repetition-time $(\mathrm{TR})=2200 \mathrm{~ms}$; echo-time $(\mathrm{TE})=3.05 \mathrm{~ms}$; inversion-time $=1100$ ms; flip-angle $=10^{\circ}$; matrix size $=256 \times 256$; field-of-view $(\mathrm{FOV})=220 \times$ $220 \mathrm{~mm}$; slice thickness $=1.0 \mathrm{~mm}]$. Proton-density $(\mathrm{PD})$ and T2-weighted images were collected, using a dual-echo turbo spin-echo pulse sequence $\left(\mathrm{TR}=8000 \mathrm{~ms}\right.$; TE1, $2=17,133 \mathrm{~ms}$; flip-angle $=150^{\circ}$; matrix size $=$ $256 \times 256 ; \mathrm{FOV}=240 \times 240 \mathrm{~mm}$; slice thickness $=5.0 \mathrm{~mm}$; turbo factor $=5$ ). DTI was performed in the axial plane, using a single-shot spin-echo echo-planar pulse sequence $(\mathrm{TR}=10,000 \mathrm{~ms} ; \mathrm{TE}=87 \mathrm{~ms}$; flip-angle $=90^{\circ} ;$ matrix size $=128 \times 128 ;$ FOV $=220 \times 220 \mathrm{~mm}$; thickness $=1.9 \mathrm{~mm}$; slices $=75$; no interslice gap; diffusion gradient directions $=12 ; \mathrm{b}=0,50,700 \mathrm{~s} / \mathrm{mm}^{2}$ ). The parallel imaging technique, generalized-autocalibrating-partially-parallel-acquisition (GRAPPA) with an acceleration factor of two was used to accelerate imaging and reduce distortion. Two DTI series were collected from each subject for subsequent averaging to improve signal-to-noise.

Data processing. Brain images of all subjects, including PD and T2weighted, and T1-weighted images were evaluated for lesions. One control subject showed a cerebellar cyst, which would contaminate control axial and radial diffusivity values of the surrounding tissue, and was excluded, leaving 12 CCHS and 26 control subjects. Diffusion-weighted and b0 images were also examined for motion and other artifacts; all remaining subjects were included.

Data were processed using the statistical parametric mapping package SPM5 (http://www.fil.ion.ucl.ac.uk/spm/software/spm5/), DTI-Studio (http:// lbam.med.jhmi.edu/), and Matlab-based (The MathWorks Inc, Natick, MA) custom software.

Calculation of axial and radial diffusivity. Using diffusion-weighted and b0 images, the average background noise level outside the brain parenchyma was calculated, providing a minimum threshold to mask nonbrain regions. Using DTI-Studio, diffusion tensors were calculated from b0 images and diffusion-weighted images (50 and $\left.700 \mathrm{~s} / \mathrm{mm}^{2}\right)$. Principal eigenvalues $(\lambda 1, \lambda 2$, and $\lambda 3$ ) were derived by diagonalizing the diffusion tensor at each voxel. Axial $\left(\lambda_{\|}=\lambda 1\right)$, and radial diffusivity $\left[\lambda_{\perp}=(\lambda 2+\lambda 3) / 2\right]$ maps were derived from each DTI series (12), in which voxel intensities represent the corresponding diffusivity values.

Data analysis. For each subject, the two axial and radial diffusivity maps derived from two separate DTI series were realigned to account for possible motion, and averaged to create one axial and radial diffusivity map. Similarly, b0 images from the two DTI series were realigned, and averaged to create one b0 image per subject. The averaged axial and radial diffusivity maps were used for subsequent voxel-based-analyses, and b0 images for normalization.

Voxel-based-analyses. We used voxel-based-analysis procedures, which allow comparisons of values voxel-by-voxel between groups. Axial and radial diffusivity maps were normalized to Montreal Neurologic Institute (MNI) space. T2-weighted images (b0 images) of each subject were normalized to the MNI space, using a priori-defined distributions of tissue types, and the resulting normalization parameters were applied to axial and radial diffusivity maps. The normalized maps were smoothed using a Gaussian filter with full-width-at-half-maximum $=10 \mathrm{~mm}$. The normalized and smoothed axial and radial diffusivity maps were compared voxel-by-voxel between groups using analysis of covariance, with age and gender as covariates (false discovery rate correction for multiple comparisons, $p<0.05$ ).

High-resolution T1-weighted images from one control subject were also normalized to MNI space, and used as background images for structural identification. Statistical parametric maps that identified significant group differences in axial and radial diffusivity in brainstem and cerebellar regions were created for regions showing increased axial diffusivity only, increased radial diffusivity only, and both increased axial and radial diffusivity, and overlaid onto background images. Diencephalic and forebrain deficits were suppressed to focus on brainstem and cerebellar regions.
Region-of-interest analyses. Regions-of-interest were created for clusters showing increased axial diffusivity only, radial diffusivity alone, and both increased axial and radial diffusivity in brainstem and cerebellar regions, based on voxel-based-analyses procedures for all distinct locations. Using these region-of-interest masks, mean axial and radial diffusivity values were derived from each individual's normalized and smoothed axial and radial diffusivity maps, and significant group differences for these distinct brain locations were evaluated using multivariate analysis of covariance (SPSS, v15, Chicago, IL), with age and gender included as covariates.

Comparison of structural injury and earlier-described functional deficits. We had earlier performed hypercapnic $\left(5 \% \mathrm{CO}_{2}+95 \% \mathrm{O}_{2}\right)$, hypoxic $\left(15 \% \mathrm{O}_{2}+85 \% \mathrm{~N}_{2}\right)$, and cold pressor (forehead) challenges in CCHS children during functional MRI (9-11). Thirteen to 14 CCHS and 14 control subjects were examined with a conventional blood-oxygen-level-dependent MRI protocol; a baseline series was collected, followed by challenges (hypercapnia, hypoxia, and cold pressor) with 10 min rest intervals. The functional series were evaluated with cluster and volume-of-interest analyses (9-11). Clusters showing functional deficits between groups were compared with structural injuries found here.

\section{RESULTS}

Visual examination of T1-, T2-, and PD-weighted images from CCHS subjects showed no obvious abnormalities in brainstem and cerebellar regions. However, axial and radial diffusivity measures showed multiple brainstem and cerebellar sites with higher values in CCHS over control subjects (Figs. 1 and 2). No regions showed significantly reduced axial or radial diffusivity values in CCHS versus control subjects.

Mean axial diffusivity values for brain regions showing significant group differences are summarized in Table 1. Clusters with injury, indicated by increased axial diffusivity, with no significant change in radial diffusivity extended from the dorsal midbrain on the midline, caudally through the periaqueductal gray, oculomotor nuclei, raphé, and decussation of the superior cerebellar peduncle (Fig. $1 a, d, j$ ), then ventrally to the mid-pons toward the basal-pons (Fig. 1b, g, $k$ ), ending in the midline of the caudal basal-pons (Fig. 1c, $l$ ). Additional areas within lateral portions, bilaterally, of the crus cerebri, extending to the hippocampus and amygdala (Fig. 1e, $f, h, i$ ), and right ventrolateral medulla (Fig. $1 m, n, o$ ) also showed higher axial diffusivity.

Mean radial diffusivity values for brain sites that show significant group differences are summarized in Table 1. Regions with higher radial diffusivity and no significant change in axial diffusivity in CCHS emerged in the bilateral superior (Fig. $2 a, b, d, e, g, i)$ and inferior cerebellar peduncles (Fig. $2 f$, $k, l$ ), bilateral regions within the cerebellar cortex (Fig. $2 c, h$, $n, o$ ) and deep nuclei (Fig. 2j, $m$ ).

Clusters of both increased axial and radial diffusivity appeared in caudal and ventrolateral portions of the cerebellar cortices, bordering deep nuclei, and in the pretectal area.

The structural injuries found here showed considerable correspondence to previously described functional deficits $(9-11)$ in brainstem and cerebellar areas to hypercapnic, hypoxic, and cold pressor challenges in a different CCHS patient population (Fig. 3).

\section{DISCUSSION}

Visual examination of routine MR images from individual CCHS subjects did not show brainstem injury sufficient to explain CCHS characteristics. However, microstructural 


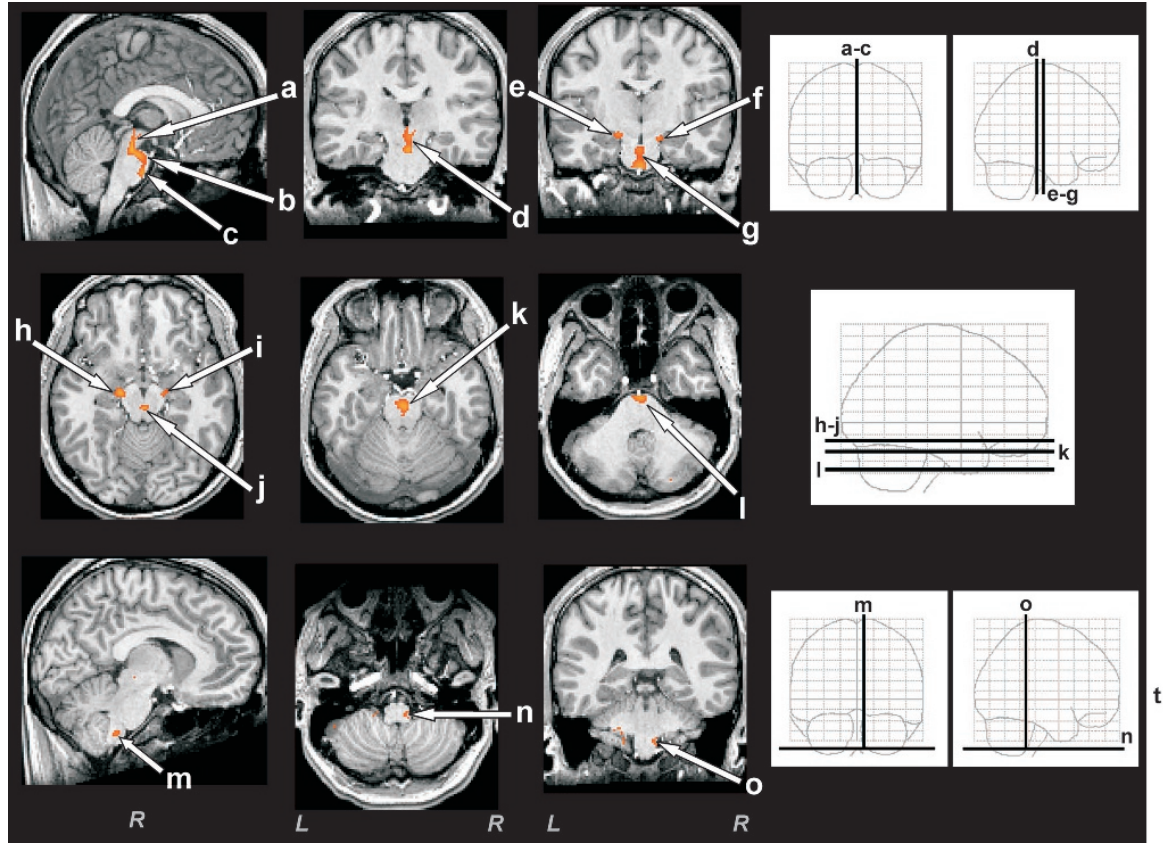

5
4
3
2
1
0
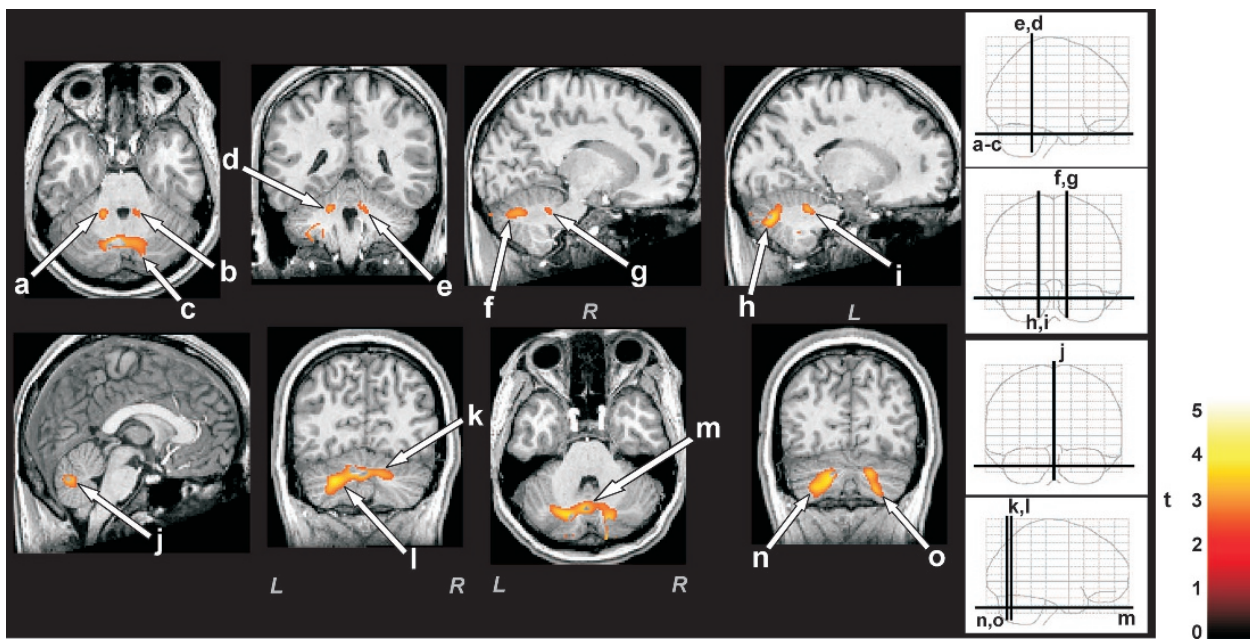

Figure 1. Brainstem areas with significantly increased axial diffusivity only. Abnormal clusters appeared in the dorsal midbrain on the midline, caudally through the periaqueductal gray, oculomotor nuclei and dorsal and central raphé and the decussation of the superior cerebellar peduncle $(a, d, j)$, ventrally to the mid-pons toward the basal-pons $(b, g, k)$, ending in the midline of the caudal basal pons $(c, l)$. Other abnormal brain areas emerged in bilateral crus cerebri, extending to the hippocampus and amygdala $(e, f, h, i)$, and right lateral medulla $(m, n, o)$. All images are in neurologic convention $(L=L e f t$, $R=R i g h t)$, and color scale represents t-statistic values $(p<0.05$, false discovery rate correction for multiple comparisons).

Figure 2. Cerebellar sites with significantly increased radial diffusivity only. Regions with significant deficits emerged in the bilateral superior $(a, b, d, e, g, i)$ and inferior cerebellar peduncles $(f, k, l)$, bilateral cerebellar cortices $(c, h, n, o)$ and deep nuclei $(j, m)$. Figure conventions are the same as in Figure 1. changes determined by quantitative axial and radial diffusivity measures were remarkable in CCHS. Diffusion abnormalities appeared in the midbrain, cerebral crus, pons, lateral medulla, the cerebellum, decussating midbrain superior cerebellar and pontine cerebellar axons, and superior and inferior cerebellar peduncles. Several regions with abnormal diffusivity values overlapped sites that previously showed functional deficits to hypercapnic, hypoxic, and cold pressor challenges (9-11). More-rostral areas, e.g., hippocampus, basal ganglia, also showed abnormal diffusivity, but the focus here was on brainstem and cerebellar regions. Left unaffected were large components of the pyramidal tract. Abnormal diffusivity was more prominent in medial, rather than lateral portions of the brainstem, except for one lateral region in the medulla. Abnormal medial sites included the periaqueductal gray, oculomotor nuclei and midbrain raphé that help regulate multiple motor, breathing and autonomic control processes.

Changes in axial and radial diffusivity. Abnormal brain regions show either radial or axial diffusivity changes or changes in both. Radial diffusivity measures motion of water molecules perpendicular to fibers, and changes primarily indicate myelin alterations. Increased radial diffusivity is usually interpreted as abnormalities in myelinated membranes surrounding axons in shiverer mice $(12,14)$; such mice form axon bundles with significantly less myelin compared with controls. These mice show increased radial diffusivity with minimal changes in axial diffusivity, suggesting that increased radial diffusivity without altered axial diffusivity results from reduced or absent myelin. The condition leads to increased freedom of crossfiber diffusion that allows faster motion of water molecules perpendicular to tissue fibers.

Myelin abnormalities could result from a failure to form myelin (dysmyelination) e.g., the shiverer mouse, or to demyelination from injury, such as hypoxic events in CCHS. Radial diffusivity increases in a cuprizone animal model of demyelination (a neurotoxin used to study demyelination) (15), with axial diffusivity comparable to controls in sub-acute stages. Abnormalities in myelin-supporting oligodendrocytes, which 
Table 1. Brain regions with significantly increased axial or radial diffusivity values in CCHS compared to control subjects. CCHS and control group mean values across all distinct locations in brainstem and cerebellar areas are presented with standard deviations. Some groups of clusters encompass multiple brain sites

\begin{tabular}{|c|c|c|c|c|c|c|}
\hline \multirow[b]{2}{*}{ Measures } & \multirow[b]{2}{*}{ Regions } & \multicolumn{2}{|c|}{ Subjects } & \multirow[b]{2}{*}{$\begin{array}{l}\text { Voxels } \\
\left(3.4 \mathrm{~mm}^{3}\right)\end{array}$} & \multirow[b]{2}{*}{$p$} & \multirow[b]{2}{*}{ Figures } \\
\hline & & $\begin{array}{c}\text { CCHS } \\
\text { (Mean } \pm \mathrm{SD})\end{array}$ & $\begin{array}{c}\text { Controls } \\
(\text { Mean } \pm \text { SD })\end{array}$ & & & \\
\hline \multirow[t]{4}{*}{$\begin{array}{l}\text { Axial diffusivity only } \\
\qquad\left(\times 10^{-3} \mathrm{~mm}^{2} / \mathrm{s}\right)\end{array}$} & $\begin{array}{l}\text { Dorsal midbrain, extending to periaqueductal } \\
\text { gray, dorsal and central raphé, through } \\
\text { superior cerebellar decussation, and to mid } \\
\text { basilar pons }\end{array}$ & $1.35 \pm 0.08$ & $1.25 \pm 0.08$ & 378 & 0.011 & $1 a-d, g, j-l$ \\
\hline & $\begin{array}{l}\text { Left crus cerebri extending to hippocampus } \\
\text { and amygdala }\end{array}$ & $1.19 \pm 0.06$ & $1.12 \pm 0.06$ & 75 & 0.012 & $1 e, h$ \\
\hline & $\begin{array}{l}\text { Right crus cerebri extending to hippocampus } \\
\text { and amygdala }\end{array}$ & $1.30 \pm 0.06$ & $1.22 \pm 0.08$ & 57 & 0.017 & $1 f, i$ \\
\hline & Right ventrolateral medulla & $1.98 \pm 0.12$ & $1.79 \pm 0.20$ & 26 & 0.009 & $1 m-o$ \\
\hline \multirow{5}{*}{$\begin{array}{l}\text { Radial diffusivity only } \\
\qquad\left(\times 10^{-3} \mathrm{~mm}^{2} / \mathrm{s}\right)\end{array}$} & Left superior cerebellar peduncle & $0.40 \pm 0.03$ & $0.37 \pm 0.02$ & 101 & 0.029 & $2 a, d, i$ \\
\hline & Right superior cerebellar peduncle & $0.63 \pm 0.11$ & $0.56 \pm 0.06$ & 60 & 0.033 & $2 b, e, g$ \\
\hline & $\begin{array}{l}\text { Bilateral inferior cerebellar peduncles } \\
\text { extending to deep nuclei }\end{array}$ & $0.41 \pm 0.02$ & $0.39 \pm 0.01$ & 1316 & 0.007 & $2 f, j-m$ \\
\hline & Left cerebellar cortex & $0.38 \pm 0.02$ & $0.36 \pm 0.02$ & 558 & 0.006 & $2 h, n$ \\
\hline & Right cerebellar cortex & $0.40 \pm 0.02$ & $0.38 \pm 0.02$ & 347 & 0.003 & $2 c, o$ \\
\hline \multirow{5}{*}{$\begin{array}{l}\text { Axial diffusivity } \\
\qquad\left(\times 10^{-3} \mathrm{~mm}^{2} / \mathrm{s}\right)\end{array}$} & Cerebellar deep nuclei & $0.71 \pm 0.04$ & $0.68 \pm 0.03$ & 38 & 0.031 & \\
\hline & Left caudal cerebral cortex & $0.75 \pm 0.11$ & $0.66 \pm 0.06$ & 67 & 0.021 & \\
\hline & Right caudal cerebral cortex & $0.67 \pm 0.04$ & $0.63 \pm 0.02$ & 232 & 0.005 & \\
\hline & Left ventral cerebral cortex & $1.10 \pm 0.11$ & $0.97 \pm 0.09$ & 318 & 0.006 & \\
\hline & Pretectal area & $1.14 \pm 0.11$ & $1.04 \pm 0.09$ & 75 & 0.010 & \\
\hline \multirow{5}{*}{$\begin{array}{l}\text { Radial diffusivity } \\
\qquad\left(\times 10^{-3} \mathrm{~mm}^{2} / \mathrm{s}\right)\end{array}$} & Cerebellar deep nuclei & $0.47 \pm 0.02$ & $0.44 \pm 0.03$ & 38 & 0.010 & \\
\hline & Left caudal cerebral cortex & $0.44 \pm 0.05$ & $0.38 \pm 0.04$ & 67 & 0.010 & \\
\hline & Right caudal cerebral cortex & $0.43 \pm 0.03$ & $0.40 \pm 0.02$ & 232 & 0.006 & \\
\hline & Left ventral cerebral cortex & $0.63 \pm 0.06$ & $0.55 \pm 0.06$ & 318 & 0.004 & \\
\hline & Pretectal area & $0.64 \pm 0.06$ & $0.57 \pm 0.07$ & 75 & 0.020 & \\
\hline
\end{tabular}

CCHS, Congenital central hypoventilation syndrome.

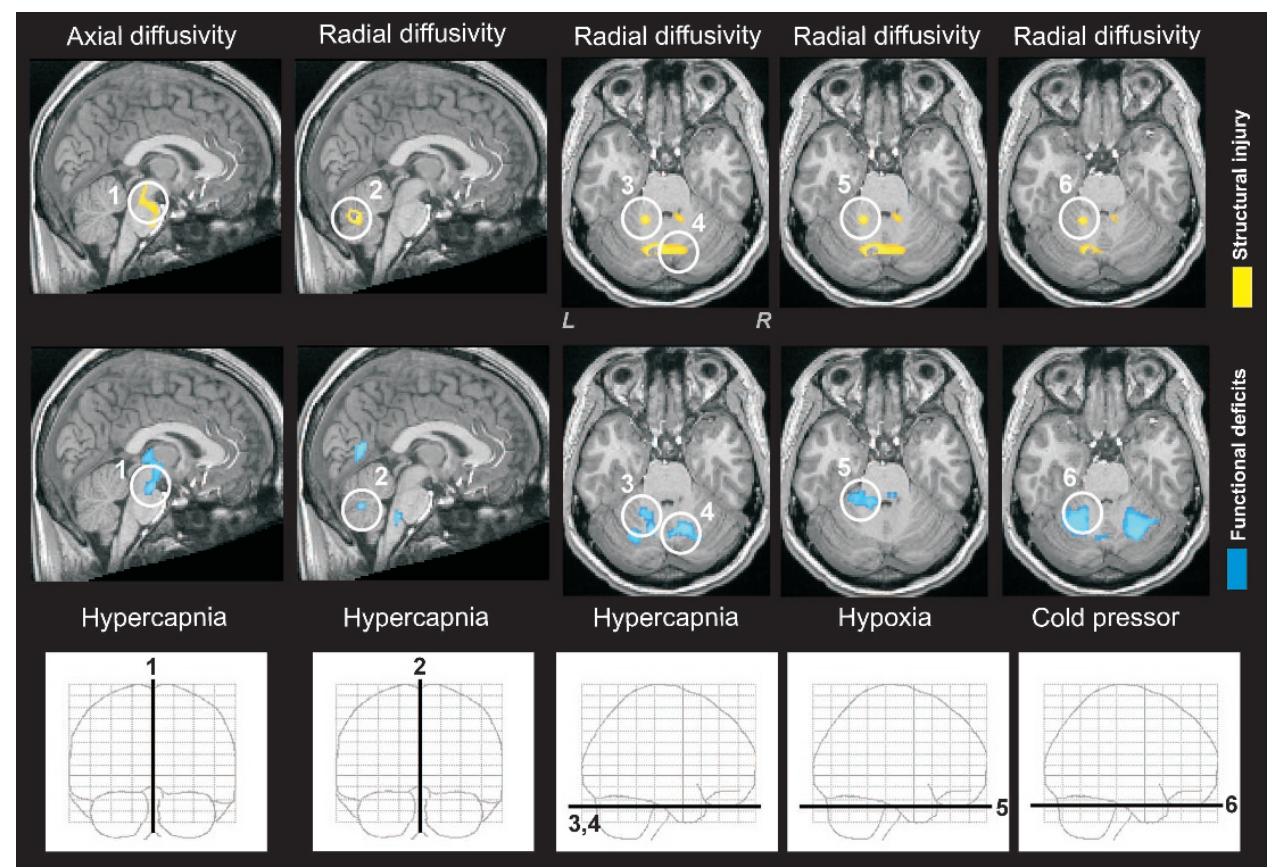

Figure 3. Structural injury overlapped some of the previously reported functional deficits in another group of CCHS subjects. The upper panels show selected regions of structural injury (yellow) based on axial and radial diffusivity measures, and the lower panels show sites of functional deficits (blue) to hypercapnic (adapted from, Harper RM et al., J Neurophysiol. 93:1647-1658, Copyright (C) 2005 The American Physiologic Society, with permission), hypoxic, and cold pressor (adapted from, Macey PM et al., Pediatr Res 57:500-509, Copyright (C) 2005 International Pediatric Research Foundation) challenges. Comparable areas are indicated by circles, and numbers indicate overlapping structural and functional deficits.

are susceptible to hypoxia, may alter myelin, and hence, radial diffusivity changes (16). Thus, significantly increased radial diffusivity alone implies reduced myelin from abnormal development or injurious processes, such as hypoxia.

Axial diffusivity measures water molecule motion parallel to axons, and changes likely result primarily from axonal abnormalities (12). Increased radial and axial diffusivity appears in trans callosal fibers in multiple sclerosis (17), and patients with traumatic brain injury (18), which has both myelin and axonal damage, and in brain regions with primary infarcts following stroke (19). However, no histopathological model currently exists analogous to the shiverer mouse show- 
ing only increased axial diffusivity without significant radial diffusivity changes. Axial diffusivity can significantly increase with increased extra-axonal space resulting from reduced axonal density or axonal caliber, which allows faster water molecule movement parallel to axons. Axonal damage found here may derive from inadequate development, as well as processes secondary to hypoxia, leading to reduced axonal density or caliber. However, axonal alterations secondary to hypoxia are less likely; significantly increased radial diffusivity appears before increased axial radial diffusivity emerges (13). If comparable injury occurs in human CCHS brainstem areas, the increased axial diffusivity likely develops from developmental injury. In animal models, both peripheral and central autonomic nuclei are affected by Phox $2 \mathrm{~b}$ mutation (20), and certain brainstem autonomic nuclei are located in affected midbrain areas found here.

Midbrain and pontine abnormalities. Affected structures within the midbrain and pons influence several physiologic characteristics that are deficient in CCHS. The injured areas included the oculomotor and Edinger-Westphal parasympathetic nuclei, which mediate eye movement and ciliary nerve parasympathetic action, respectively, the latter reflected in altered pupillary constriction found in CCHS $(3,21)$, and the former expressed as impaired eye movements. Abnormal diffusivity values also appeared in portions of the dorsal and central raphé, a principally-serotonergic structure showing sleep-state action (22). Serotonergic neurons modify upper airway muscle tone, participate in regulation of vascular activity, and may mediate chemosensitive responses (23-25). Localized clusters of abnormal diffusivity in the midbrain overlapped areas of functional deficits found earlier to $\mathrm{CO}_{2}$ challenges (9). The periaqueductal gray, which projects to the rostral ventrolateral medulla (26) as well as to the solitary tract, dorsal vagal and retroambiguus nuclei (27), thus participating in blood pressure and breathing control, showed injury. The nearby decussating midbrain superior cerebellar peduncle fibers were also damaged. The pontine and midbrain injuries likely contribute significantly to state-related respiratory and autonomic deficiencies in CCHS.

Cerebellar abnormalities. A portion of the affected cerebellar cortical sites, lying within the vermis, has long been associated with cardiovascular and breathing coordination (28). The cerebellum dampens extremes of blood pressure change (7), and coordinates cardiovascular and sympathetic responses to somatic motor efforts (29). The deep cerebellar fastigial nuclei are chemosensitive, and play critical roles in ventilation (30). The cerebellar cortex and fastigial nuclei respond markedly to a $5 \% \mathrm{CO}_{2}$ challenge in normal subjects (9), responses that are impaired in CCHS (9). Functional deficits to $\mathrm{CO}_{2}$ and cold pressor challenges in the cerebellar cortex $(9,10)$, and hypoxic response deficits (11) to cerebellar axonal areas overlap structural injury found here (Fig. 3).

Although the cerebellum is not targeted in Phox $2 \mathrm{~b}$ knockout models, other processes may be operating. Serotonergic nerve fibers project throughout the cerebellum, including the deep nuclei (31), and these nerve fibers may be absent or injured in CCHS through damage to the dorsal and central raphé appearing here. PHOX2B plays a significant role in differentiation of central serotonergic neurons (32,33); the injury to serotonergic neurons of the raphé suggests a direct effect on specific regions of the 5-HT system.

Cerebellar Purkinje cells inhibit neurons in the fastigial nuclei, which project to sympathetic nervous system regulatory sites in the brainstem and rostral brain. Injury to the fastigial nuclei likely contributes to a characteristic of CCHS, impaired blood pressure regulation, including an enhanced propensity for syncope, and absence of nocturnal "dipping" (34).

Forebrain areas. We did not include forebrain structural injuries here, since the focus was on brainstem and cerebellar areas. We earlier described forebrain changes using T2relaxometry and mean-diffusivity measures in these same CCHS patients $(4,5)$; these sites included the basal ganglia, basal forebrain, occipital, and temporal cortices.

Axonal pathways. Injuries in major fiber bundles were remarkable in their specificity. The cerebral crus was affected bilaterally, but only in discrete areas of the topographicallyorganized lateral portion of the crus that carries fibers from the temporal, parietal, and occipital cortex to the pons (35). Abnormal axons from the temporal cortex may contribute to affective symptoms in CCHS (36). No significant abnormalities were found in the medullary pyramidal tract, a finding consistent with the absence of cortico-spinal upper motoneuron signs in CCHS.

Damage appeared in the superior and inferior cerebellar peduncles, likely adding to the cerebellar sympathetic and respiratory regulatory deficits described earlier. The superior cerebellar peduncles include deep cerebellar nuclei fibers, and coordinate motor action, including respiratory motor action, through the red nucleus, midbrain and thalamus (37). Inferior cerebellar peduncle fibers link Purkinje and deep nuclei neurons with vestibular nuclei, inferior olive, and medullary sympathetic areas (38), interfaces essential for blood pressure responses to body movement (39). Injured axons here may interfere with sympathetic outflow, such as syncope from rapid body position movement, a common characteristic in CCHS.

Medullary sites. A portion of the right lateral medulla showed increased axial diffusivity. The area overlaps a region previously implicated in $\mathrm{CO}_{2}$ insensitivity and impaired breathing during sleep in adults, the paragigantocellularis lateralis (40). The nucleus of the solitary tract (NTS), an essential component in baroreceptor integration and chemosensitivity, develops improperly in Phox $2 \mathrm{~b}$ knockout models (41), and showed impaired functional responses to hypercapnic challenges in CCHS (9). However, neither axial nor radial diffusivity measures showed NTS structural injury here. Several possibilities emerge for this apparent contradiction to the mouse anatomical and human functional studies. The technique used here may have insufficient spatial resolution or sensitivity to adequately detect injury, damage may occur elsewhere in peripheral or other autonomic ganglia in humans to provide functional deficits with no structural injury to the NTS, or the mouse model may not precisely parallel the human condition.

Limitations. Limitations include the restricted image spatial resolution and analyses procedures. Spatial resolution is imperfect with partial volume effects, as well as voxel-based 
analysis procedures, which require normalizing each subject's brain volume to a standard common space. Since such normalization may be imprecise in all subjects, the findings are only accurate to within a few millimeters, depending on brain location and degree of smoothing. Diffusion tensor images are prone to distortion in brain areas close to bone/CSF, such as the frontal lobes. However, use of the GRAPPA parallel imaging technique substantially reduced image distortions and susceptibility artifacts.

In conclusion, the brainstem in CCHS children shows abnormal axial diffusion characteristics with clusters extending from the rostral dorsal midbrain through the periaqueductal gray and midline raphé, oculomotor nuclei, and decussating superior cerebellar fibers to the basilar pons, as well as the cerebral crus and the lateral medulla. Injury appears in the superior and inferior cerebellar peduncles, and in vermal and lateral regions of the cerebellar cortex and deep nuclei, as indicated by radial diffusivity. The extensive injury to decussating cerebellar fibers in the midbrain and pons, as well as in the cerebellum could participate in a range of state-dependent integrative autonomic and motor functions impaired in CCHS. The eye movement and autonomic characteristics of the syndrome may derive from the oculomotor and raphé injury found here. Some brain regions with structural injury overlapped areas with previously identified functional deficits to hypercapnic, hypoxic, and cold pressor challenges in CCHS. Other sites, expected to be injured from animal models of Phox $2 b$, such as the NTS, showed no structural damage.

Acknowledgments. We thank Ms. Rebecca Harper and Ms. Annaise Magliore for assistance with data collection.

\section{REFERENCES}

1. Haddad GG, Mazza NM, Defendini R, Blanc WA, Driscoll JM, Epstein MA, Epstein RA, Mellins RB 1978 Congenital failure of automatic control of ventilation, gastrointestinal motility and heart rate. Medicine (Baltimore) 57:517-526

2. Paton JY, Swaminathan S, Sargent CW, Keens TG 1989 Hypoxic and hypercapnic ventilatory responses in awake children with congenital central hypoventilation syndrome. Am Rev Respir Dis 140:368-372

3. 1999 Idiopathic congenital central hypoventilation syndrome: diagnosis and management. American Thoracic Society. Am J Respir Crit Care Med 160:368-373

4. Kumar R, Macey PM, Woo MA, Alger JR, Harper RM 2006 Elevated mean diffusivity in widespread brain regions in congenital central hypoventilation syndrome. J Magn Reson Imaging 24:1252-1258

5. Kumar R, Macey PM, Woo MA, Alger JR, Keens TG, Harper RM 2005 Neuroanatomic deficits in congenital central hypoventilation syndrome. J Comp Neurol 487:361-371

6. Amiel J, Laudier B, Attie-Bitach T, Trang H, de Pontual L, Gener B, Trochet D, Etchevers H, Ray P, Simonneau M, Vekemans M, Munnich A, Gaultier C, Lyonnet S 2003 Polyalanine expansion and frameshift mutations of the paired-like homeobox gene PHOX2B in congenital central hypoventilation syndrome. Nat Genet 33:459-461

7. Lutherer LO, Lutherer BC, Dormer KJ, Janssen HF, Barnes CD 1983 Bilateral lesions of the fastigial nucleus prevent the recovery of blood pressure following hypotension induced by hemorrhage or administration of endotoxin. Brain Res 269:251-257

8. Kerman IA, Enquist LW, Watson SJ, Yates BJ 2003 Brainstem substrates of sympatho-motor circuitry identified using trans-synaptic tracing with pseudorabies virus recombinants. J Neurosci 23:4657-4666

9. Harper RM, Macey PM, Woo MA, Macey KE, Keens TG, Gozal D, Alger JR 2005 Hypercapnic exposure in congenital central hypoventilation syndrome reveals CNS respiratory control mechanisms. J Neurophysiol 93:1647-1658

10. Macey PM, Macey KE, Woo MA, Keens TG, Harper RM 2005 Aberrant neural responses to cold pressor challenges in congenital central hypoventilation syndrome. Pediatr Res 57:500-509
11. Macey PM, Woo MA, Macey KE, Keens TG, Saeed MM, Alger JR, Harper RM 2005 Hypoxia reveals posterior thalamic, cerebellar, midbrain, and limbic deficits in congenital central hypoventilation syndrome. J Appl Physiol 98:958-969

12. Song SK, Sun SW, Ramsbottom MJ, Chang C, Russell J, Cross AH 2002 Dysmyelination revealed through MRI as increased radial (but unchanged axial) diffusion of water. Neuroimage 17:1429-1436

13. Sun SW, Liang HF, Le TQ, Armstrong RC, Cross AH, Song SK 2006 Differential sensitivity of in vivo and ex vivo diffusion tensor imaging to evolving optic nerve injury in mice with retinal ischemia. Neuroimage 32:1195-1204

14. Nair G, Tanahashi Y, Low HP, Billings-Gagliardi S, Schwartz WJ, Duong TQ 2005 Myelination and long diffusion times alter diffusion-tensor-imaging contrast in myelin-deficient shiverer mice. Neuroimage 28:165-174

15. Song SK, Yoshino J, Le TQ, Lin SJ, Sun SW, Cross AH, Armstrong RC 2005 Demyelination increases radial diffusivity in corpus callosum of mouse brain. Neuroimage 26:132-140

16. Harsan LA, Poulet P, Guignard B, Parizel N, Skoff RP, Ghandour MS 2007 Astrocytic hypertrophy in dysmyelination influences the diffusion anisotropy of white matter. J Neurosci Res 85:935-944

17. Lowe MJ, Horenstein C, Hirsch JG, Marrie RA, Stone L, Bhattacharyya PK, Gass A, Phillips MD 2006 Functional pathway-defined MRI diffusion measures reveal increased transverse diffusivity of water in multiple sclerosis. Neuroimage 32:1127-1133

18. Kraus MF, Susmaras T, Caughlin BP, Walker CJ, Sweeney JA, Little DM 2007 White matter integrity and cognition in chronic traumatic brain injury: a diffusion tensor imaging study. Brain 130:2508-2519

19. Pierpaoli C, Barnett A, Pajevic S, Chen R, Penix LR, Virta A, Basser P 2001 Water diffusion changes in Wallerian degeneration and their dependence on white matter architecture. Neuroimage 13:1174-1185

20. Dauger S, Pattyn A, Lofaso F, Gaultier C, Goridis C, Gallego J, Brunet JF 2003 Phox $2 \mathrm{~b}$ controls the development of peripheral chemoreceptors and afferent visceral pathways. Development 130:6635-6642

21. Warwick R 1954 The ocular parasympathetic nerve supply and its mesencephalic sources. J Anat 88:71-93

22. McGinty DJ, Harper RM 1976 Dorsal raphe neurons: depression of firing during sleep in cats. Brain Res 101:569-575

23. Fenik VB, Davies RO, Kubin L 2005 REM sleep-like atonia of hypoglossal (XII) motoneurons is caused by loss of noradrenergic and serotonergic inputs. Am J Respir Crit Care Med 172:1322-1330

24. Wang W, Pizzonia JH, Richerson GB 1998 Chemosensitivity of rat medullary raphe neurones in primary tissue culture. J Physiol 511:433-450

25. Goadsby PJ, Piper RD, Lambert GA, Lance JW 1985 Effect of stimulation of nucleus raphe dorsalis on carotid blood flow. II. The cat. Am J Physiol 248:R263-R269

26. Carrive P, Bandler R, Dampney RA 1988 Anatomical evidence that hypertension associated with the defence reaction in the cat is mediated by a direct projection from a restricted portion of the midbrain periaqueductal grey to the subretrofacial nucleus of the medulla. Brain Res 460:339-345

27. Cowie RJ, Holstege G 1992 Dorsal mesencephalic projections to pons, medulla, and spinal cord in the cat: limbic and non-limbic components. J Comp Neurol 319:536-559

28. Moruzzi G 1950 The cerebellar influence in the autonomic sphere. In: Problems in Cerebellar Physiology. Charles C Thomas, Springfield, pp 74-96

29. Holmes MJ, Cotter LA, Arendt HE, Cass SP, Yates BJ 2002 Effects of lesions of the caudal cerebellar vermis on cardiovascular regulation in awake cats. Brain Res 938:62-72

30. Xu F, Frazier DT 1997 Respiratory-related neurons of the fastigial nucleus in response to chemical and mechanical challenges. J Appl Physiol 82:1177-1184

31. Takeuchi Y, Kimura H, Sano Y 1982 Immunohistochemical demonstration of serotonin-containing nerve fibers in the cerebellum. Cell Tissue Res 226:1-12

32. Pattyn A, Simplicio N, van Doorninck JH, Goridis C, Guillemot F, Brunet JF 2004 Ascl1/Mash1 is required for the development of central serotonergic neurons. Nat Neurosci 7:589-595

33. Pattyn A, Vallstedt A, Dias JM, Samad OA, Krumlauf R, Rijli FM, Brunet JF, Ericson J 2003 Coordinated temporal and spatial control of motor neuron and serotonergic neuron generation from a common pool of CNS progenitors. Genes Dev 17:729-737

34. Trang H, Boureghda S, Denjoy I, Alia M, Kabaker M 2003 24-hour BP in children with congenital central hypoventilation syndrome. Chest 124:1393-1399

35. Schmahmann JD, Pandya DN 1992 Course of the fiber pathways to pons from parasensory association areas in the rhesus monkey. J Comp Neurol 326:159-179

36. Hauser P, Altshuler LL, Berrettini W, Dauphinais ID, Gelernter J, Post RM 1989 Temporal lobe measurement in primary affective disorder by magnetic resonance imaging. J Neuropsychiatry Clin Neurosci 1:128-134

37. Castro AJ 1978 Projections of the superior cerebellar peduncle in rats and the development of new connections in response to neonatal hemicerebellectomy. J Comp Neurol 178:611-627

38. Sugihara I, Wu H, Shinoda Y 1999 Morphology of single olivocerebellar axons labeled with biotinylated dextran amine in the rat. J Comp Neurol 414:131-148

39. Yates BJ 1996 Vestibular influences on the autonomic nervous system. Ann N Y Acad Sci 781:458-473

40. Morrell MJ, Heywood P, Moosavi SH, Guz A, Stevens J 1999 Unilateral focal lesions in the rostrolateral medulla influence chemosensitivity and breathing measured during wakefulness, sleep, and exercise. J Neurol Neurosurg Psychiatry 67:637-645

41. Pattyn A, Morin X, Cremer H, Goridis C, Brunet JF 1997 Expression and interactions of the two closely related homeobox genes Phox $2 a$ and Phox $2 b$ during neurogenesis. Development 124:4065-4075 